\title{
Lack of Adrenarche
}

National Cancer Institute

\section{Source}

National Cancer Institute. Lack of Adrenarche. NCI Thesaurus. Code C132059.

Absent or incomplete development of adrenal androgen-mediated secondary sexual characteristics. 